Open Access

\title{
Effects of health intervention program on cardiometabolic risk profiles from health evaluation center in Asian population: a longitudinal study and propensity analysis
}

\author{
Chuan-Chuan Liu ${ }^{1,2^{*}}$, Chung-Lieh Hung ${ }^{1,3}$, Shou-Chuan Shih' ${ }^{4}$, Hung-Ju Ko ${ }^{2}$ and Ray-E Chang ${ }^{1 *}$
}

\begin{abstract}
Background: Health intervention program (HIP) based on diet and lifestyle modifications had been shown to improve cardiovascular risks. The effects of such program on a variety of cardiometabolic outcome measures conducted in a strict analysis remained relatively unexplored.

Materials and methods: A total of 2,660 participants (mean age: $43.3 \pm 10$ years, $63.6 \%$ male) underwent annual health survey from our health evaluation department. We implemented health intervention program (HIP) in which diet and lifestyle modifications including smoking cessation and advised physical activities were introduced. We further studied the effects of HIP on several cardiometabolic outcome measures including Framingham, metabolic scores and renal function in terms of Egfr with a mean follow-up period of 38.5 months. Propensity score (PS) matching (HIP vs non-HIP group) was used to avoid effects of case selection bias.

Results: Totally 1,004 (502 subjects for each group) left after PS matching protocol (both HIP and non-HIP group). The HIP group showed significant decline of waist circumference $(-1.46 \pm 0.61, p=0.016)$, post-prandial glucose $(-6.77 \pm 2.06, p=0.001)$, and total cholesterol level $(-4.42 \pm 2.15, p=0.04)$, with borderline increase in eGFR $(1.72 \pm 0.94, p=0.068)$ after an average of $1.91 \pm 1.14$ year follow up period. Exercise behavior significantly increased for those who received HIP when compared to the non-HIP group (44.6 vs $52.4 \%, p=0.014)$ ). PS matching and difference-in-difference (DID) analysis further confirmed the beneficial effects of ATP III reduction by HIP $(-0.36 \pm 0.06, p<0.05)$.

Conclusion: We demonstrated in our study that several cardiometabolic profiles can be substantially improved after health intervention introduction at the health evaluation center, supporting the beneficial evidence of such health intervention programs implementation based on primary prevention view points.
\end{abstract}

Keywords: Health promotion, Cardiometabolic profiles, Propensity matching, Primary prevention

\section{Introduction}

The total estimated financial burden of cardiovascular disease (CVD) for 2009 had reached $\$ 475.3$ billion in the united states, [1] with the averaged lifetime cost of ischemic stroke in the US estimated to be $\$ 140,048$. The unawareness of early stage weight control or life style and dietary modification so far remained as the major causes

\footnotetext{
* Correspondence: carrie@ms1.mmh.org.tw; rchang@ntu.edu.tw

${ }^{1}$ The Institute of Health Policy and Management, College of Public Health, National Taiwan University, Taipei, Taiwan

Full list of author information is available at the end of the article
}

of subsequent development of CVD. Thus far, Framingham risk score (FRS), a fairly robust scoring system with well-validated longitudinal epidemiological data, and had been used broadly as a "gold-standard" in projecting future cardiovascular risks [1]. On the other hand, metabolic syndrome, $[2,3]$, which takes a cluster of metabolic derangements tightly linked to cardiovascular disorders with central obesity as key clinical feature, had been demonstrated to identify subject to adverse cardiovascular outcomes in the past decade [3-6]. Compared to FRS, cardiovascular risk stratification based on the 
metabolic scoring is composed of more modifiable cardiometabolic risk factors and prone to lifestyle modification and health intervention.

Health intervention programs (HIP), which actually comprised of health promotion, counseling and associated education programs adopted by several earlier healthcare organizations based on the modification of health behaviors and dietary control, is of extraordinary clinical value in disease prevention and therapeutic intervention [7-10]. Therefore, hospitals should be implemented with cost-effective primary preventive practices both for disease prevention and health promotion. The health evaluation center, an emerging new medicare unit nearest to primary and preventive medicine, should theoretically play an pivotal role to develop or to screen and provide educative care delivery for selected subjects in need. However, there remains a gap between the ideal implementation of health promotion programs and the actual efficacy in a large volume unit from real world practice.

The aim of this study was to investigate whether the health promotion implementation may lead to effective and significant effects on cardiometabolic risk profiles based on hospital health screening programs in a tertiary medical center.

\section{Methods}

\section{Study background}

In addition to the routine and traditional offer of standard physical examination, clinical information or biochemical data collection, a health intervention program (HIP) was developed and was further funded as a new component of our routine health evaluation procedure at the health evaluation center from a tertiary medical center in Northern Taipei, Taiwan in 2002. The health intervention program (HIP) from our health examination center includes the following 3 components:

1) health education guidance preceding a health evaluation; 2) establishment of health management profiles; and 3) follow-up care and tracking. Follow-up tracking is managed by health management specialists using the Health Management Information System. This system not only records all health evaluation test results, physician diagnoses, physician recommendations and dates and times of the referral appointments, but also provides a list of abnormal results and the annual health evaluation comparison for patients who had at least two consecutive visits. This system can also facilitate specialists to track and provide follow-up care. After initially running of nearly 2 years to get the system, we started to conduct a health promotion protocol in our health evaluation center.

\section{Study design}

In our current study cohort, we analyzed the effects of such HIP implemented from those individuals who underwent two consecutive health evaluations between January 2004-2009. A physician specializing in family or internal medicine provides patients with a systematic explanation of their health evaluation report on the day following examinations. The content of the report includes test results, physical examination results, specialist recommendations, a summary and explanation of the results, and appropriate health education. Follow-up visits are scheduled with prescriptions done immediately as well as the referral and registration procedures performed on the same day. For patients who require follow up visits, appointment was made by health management specialists. Subjects not eligible and precluded in our health evaluation center may include unstable clinical conditions including acute decompensated heart failure, acute coronary syndrome, acute cerebrovascular event or renal replacement therapy. We intended to invite all subjects to participate our HIP program. However, since not all subjects were willing to participate this program, we further categorize the baseline sample into two groups: those who received health promotion interventions (HIP group) and those who did not (nonHIP group). In brief, HIP programs were characterized by both nutrition-based health education on diet habit and lifestyle modification as the following items: 1 ) education provided with the essential information on modifiable, diet-related disorders including less salt intake, to minimize food/diet exposure enriched in cholesterol and sugars, and 2) further highlighted the concept of lifestyle modification including increasing exercise or physical activities, smoking cessation, optimization of alcohol use, regular daily life schedule planning or tasks management to avoid staying up late. Education was provided by individualized instruction from special trainee/ specialist in our center. A detailed study protocol and components were listed in Additional file 1: Table S1.

\section{Study subjects recruitment and flowchart}

From the initial 28,344 persons who underwent health evaluation were eligible for our analysis, 19,686 persons who either had only one health evaluation or had a very short interval between two consecutive visits that was shorter than 36 months, leading to a total of 8,658 individuals remained. A total of 6,745 persons completed the questionnaires, while the remaining 1,913 persons were excluded. After further exclusion of those due to missing data $(n=1,876)$, incomplete reports $(1,700)$, subjects with extreme values after descriptive data analysis $(n=12)$, the final cohort for this retrospective study consisted of 3,057 persons (Fig. 1). Among them, 922 subjects received HIP and 1,738 did not. The complete 


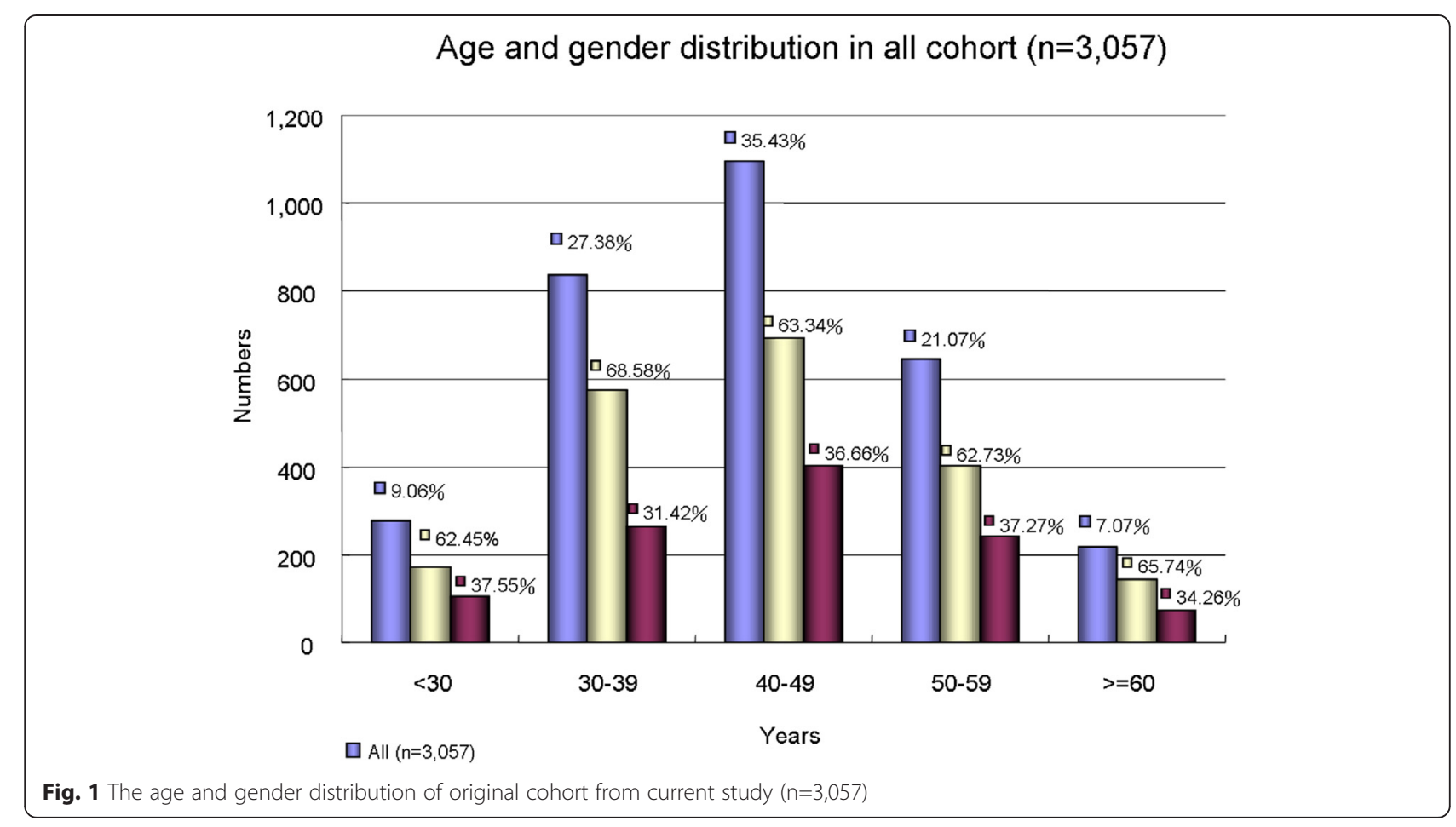

exclusion procedure of the sample is outlined in Fig. 2. The data collected from the first health evaluations constitutes the baseline, and the data collected from the second health evaluation constitutes the follow-up.

\section{Study procedure}

The recipient of a health evaluation will track his or her health status according to the contents of the report and the physician's recommendations, and will voluntarily follow-up with the department on an outpatient basis. Data considered includes physical examination questionnaires, satisfaction surveys, physiological and chemical examinations, metabolic scores (ATP III), FRS, eGFR, consultation notes from specialists, physical examination notes from physicians, records from case managers, follow-up care records, and the number of referrals and their associated costs. This study was approved by the local ethics committee of the Mackay Memorial hospital and patient information was anonymized and de-identified prior to analysis. This study received approval from the institutional review board (MMH-I-S-584) to perform retrospective research using secondary data.

\section{Outcome measures}

1. The Assessment of Metabolic Scores: ATP III risk scores

We assessed ATP III scores at baseline and at follow-up to quantify the change in health status among the intervention and control groups. A decrease in score indicates an improvement in health status, while an increase in score or no change in score indicates lack of improvement. Based on National Cholesterol Education Program-Adult Treatment Panel III (NCEP-ATP III) criteria score [11], we measured five risk indicators using a change score between 0 and 5 , using components including: 1) abdominal obesity (waist circumference $\geq 90 \mathrm{~cm}$ and $\geq 80 \mathrm{~cm}$ in men and women, respectively); 2) low high-density lipoprotein cholesterol (HDL) ( $<40 \mathrm{mg} / \mathrm{dl}$ in men, $<50 \mathrm{mg} / \mathrm{dl}$ in women); 3) high triglycerides ( $\geq 150 \mathrm{mg} / \mathrm{dl}) ; 4)$ high blood pressure (systolic blood pressure $\geq 130 \mathrm{mmHg}$ or diastolic blood pressure $\geq 85 \mathrm{mmHg}$ ); 5) and high fasting glucose $(\geq 100 \mathrm{mg} / \mathrm{dl})$. According to the NCEP-ATP III score of $0-5$, individuals with a score $\geq 3$ are categorized as having the metabolic syndrome.

2. The Assessment of Framingham Risk Score (FRS) The operational definition of the cardiovascular risk assessment for Framingham Risk Score (FRS) included indicators of cardiovascular risk established in the National Cholesterol Education Program (NCEP) guidelines: age, total cholesterol, HDL cholesterol, blood pressure, and smoking status [11]. Based on the levels of risk factors, the percentage risk of developing chronic coronary heart disease (CHD) in the subsequent 5-10 years was quantified according to the conversion table. Subsequently, the 


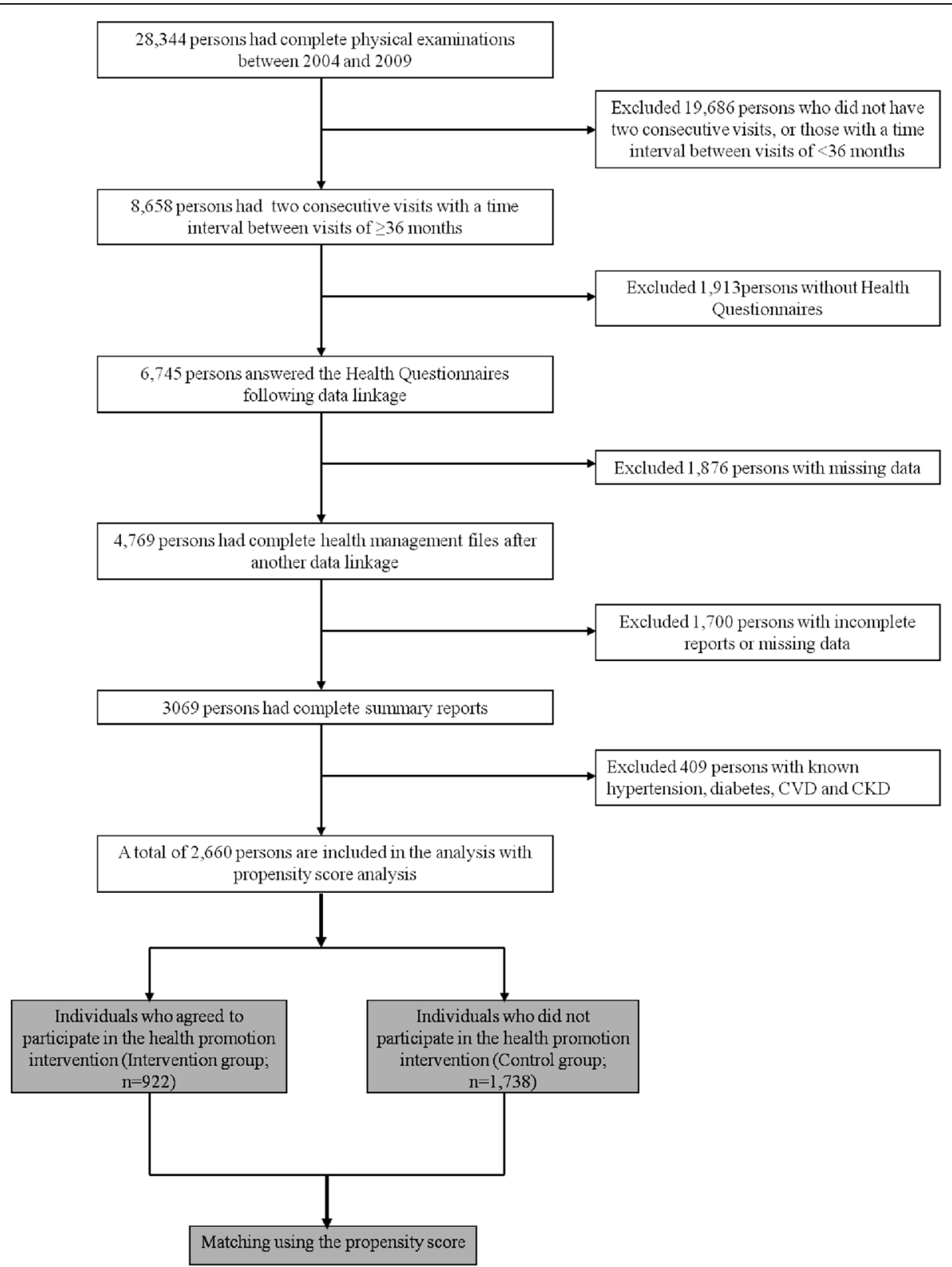

Fig. 2 The flowchart of current study subjects and subjects excluded for final analysis and propensity matching

cardiovascular risk can be delineated into high (>20\%), moderate (10-20\%), or low (<10\%) [11]. This study uses the percent change in cardiovascular risk score as the operational definition for health status improvement.

3. Estimated Glomerular Filtration Rate (eGFR) We relied on the Modification of Diet in Renal Disease (MDRD) in the definition of eGFR, which incorporated serum creatinine level into MDRD formula [12]. The percent change of eGFR from baseline to follow-up among the intervention and control groups was evaluated. An increase of eGFR score indicates health status deterioration, while a decrease indicates health status improvement.

\section{Statistical method}

The analysis comparing the health status of the intervention and control groups was completed using SPSS 17.0 statistical software. Descriptive statistical analysis including the frequency distributions and percentages for all study variables were assessed for the entire sample and also according to group (intervention versus control). 
Inferential statistical analysis of propensity score analysis was performed by logistic regression. In order to avoid selection bias of several key factors in the comparison of intervention effects between HIP and non-HIP groups, we further matched the two groups based on a propensity score. The covariates included age, gender, smoking status, drinking status, exercise status, systolic blood pressure, diastolic blood pressure, BMI, ATP III score, FRS score, eGFR, blood glucose level, cholesterol, HDL cholesterol, LDL cholesterol, triglycerides, as well as family history of hypertension, diabetes, and cardiovascular diseases. We used the caliper and the radius matching methods. In order to prove the distribution of covariates among two groups is homogeneous, the matching distance was set at 0.05 . This matching method reduces the effect of sampling bias and illustrates that the two groups consist of randomly assigned study samples. The matching algorithm for the propensity score (PS) was based on the caliper and radius matching methods, where the caliper signifies a tolerance level for the maximum distance in the propensity score. A distance of 0.05 was chosen for the current study. The use of PS ensures homogeneity in the baseline distributions of outcomes among the intervention and control groups, and also avoids estimation error caused by selection bias. Independent-samples $t$-test was used to compare the differences between the means of study continuous variables and risk indicators between groups with Chi-squared test used to evaluate proportional differences in categorical variables. Paired- $t$ test and McNemar test were used to examine continuous and categorical variable changes about health improvement in the intervention or control group from baseline to follow-up.

Difference in difference (DID) analysis was introduced in our study, which first calculated the difference in a given outcome between baseline and follow-up for each of the intervention control groups to eliminate the potential biological changes over time. Subsequently, the changes in cardiovascular risk scores are compared to estimate the differences which may reflect the difference in the improvement of health status in one group (intervention group) over the other (nonintervention group).

\section{Results}

\section{Baseline demographics}

Of the initial 3,057 individuals participated the health screen program during study period (2004-2009; Additional file 1: Figure S1), 2,660 met eligible criteria and were selected for final enrollment (Additional file 1: Figure S1). Among them, 1,691 (63.6 \%) were male and 969 (36.4 \%) were female, resulting in a male to female ratio of $1.75: 1$. As shown in Table 1, the mean \pm standard deviation (SD) age for the entire cohort was $43.3 \pm 10.0$ years, with resultant 922 and 1,738 subjects in the intervention and nonintervention groups, respectively (Additional file 1: Figure S2).

When comparing the HIP to the non-HIP group on baseline demographics, lifestyle factors, medical histories, and cardiometabolic risk scoring before PS matching, those who underwent intervention tended to be older, less male gender, higher fasting glucose, HbA1c level, total cholesterol, HDL and LDL level, higher probability of smoking behavior, and alcohol use as well as regular exercise life styles (all $p<0.05$ ). When compared the cardiometabolic risk scorings, the intervention group tended to have worse scorings when compared to the non-intervention group in terms of higher ATP III (1.49 vs 1.28 ) and lower eGFR, though the FRS did not show any significant differences between these two groups.

\section{Propensity Score (PS) analysis}

A total of 502 subjects from each group were identified and following matching showed no significant differences in any of the covariates existed between the two groups. This indicates homogeneity between the intervention and control groups following PS matching. The results of the PS are presented in Table 1 (right columns), with the association between various baseline variables and propensity score demonstrated in Table 2. The C-statistic, as estimated by ROC curve, was 0.549 . Model fitness was examined by the Hosmer and Lemeshow Goodness-of-Fit test, which indicates no significant differences between actual and model-predicted estimates $(p=0.311)$.

In brief, there seemed no major differences regarding baseline demographic data such as age, gender distribution, blood pressures (both systolic and diastolic), body mass index, waist circumference, glucose level (fasting and post-prandial), HbA1c concentration, various lipid profiles (cholesterol, triglyceride, HDL and LDL), as well as life styles (smoking, exercise and alcohol consumption behaviors) (all $\mathrm{p}$ for differences $>0.05$ ). In addition, we further demonstrated no differences of baseline cardiometabolic risk scorings including ATP III, FRS and eGFR ( $p=0.296,0.809 \& 0.704$, respectively).

\section{Baseline to follow-up differences in various clinical parameters and associated cardiometabolic risks}

Results of baseline demographic and cardiometabolic risk profiles of the $t$-test assessing differences after propensity matching at follow-up between intervention and control groups are outlined in Table 3. For both intervention and non-intervention groups, there were no major differences regarding blood pressures (both 
Table 1 Comparison of baseline data among study groups before and after propensity score matching

\begin{tabular}{|c|c|c|c|c|c|c|}
\hline \multirow[b]{2}{*}{ Variable } & \multicolumn{3}{|c|}{ Initial cohort before PSA matching $(N=2,660)$} & \multicolumn{3}{|c|}{ Cohort after PSA matching $(N=1004)$} \\
\hline & $\begin{array}{l}\text { Non-intervention } \\
(n=1,738)\end{array}$ & Intervention $(n=922)$ & $P^{a}$ & $\begin{array}{l}\text { Non-intervention } \\
(n=502)\end{array}$ & Intervention $(n=502)$ & $P^{\mathrm{a}}$ \\
\hline \multicolumn{7}{|l|}{ Demographical data } \\
\hline Age, year & $40.93(10.31)$ & $44.85(8.6)$ & $<0.001$ & $44.52(9.35)$ & $44.81(8.14)$ & 0.609 \\
\hline Male gender, \% & $65.4 \%$ & $60.2 \%$ & 0.008 & $62.9 \%$ & $64.3 \%$ & 0.646 \\
\hline \multicolumn{7}{|l|}{ Physiologic parameters } \\
\hline $\begin{array}{l}\text { Systolic blood pressure, } \\
\mathrm{mmHg}\end{array}$ & $117.32(15.04)$ & $116.68(14.55)$ & 0.293 & $117.03(13.75)$ & $117.57(14.67)$ & 0.549 \\
\hline $\begin{array}{l}\text { Diastolic blood pressure, } \\
\mathrm{mmHg}\end{array}$ & $73.43(9.81)$ & $73.45(9.84)$ & 0.946 & $73.34(9.24)$ & $73.86(9.77)$ & 0.387 \\
\hline Waist Circumference, $\mathrm{cm}$ & $80.01(10.18)$ & $80.13(9.59)$ & 0.785 & $80.5(9.3)$ & $80.5(9.8)$ & 0.99 \\
\hline Body mass index, $\mathrm{kg} / \mathrm{m}^{2}$ & $23.48(3.32)$ & $23.47(3.15)$ & 0.936 & $23.61(2.86)$ & $23.66(3.21)$ & 0.772 \\
\hline \multicolumn{7}{|l|}{ Serum sugar profiles } \\
\hline Fasting glucose, mg/dL & $92.96(13.83)$ & $94.73(15.76)$ & 0.0033 & $94.22(16.95)$ & $94.22(13.54)$ & 1.000 \\
\hline Post-prandial glucose, mg/dL & $101.51(27.92)$ & $103.73(30.92)$ & 0.139 & $104.16(33.06)$ & $103.75(28.91)$ & 0.846 \\
\hline $\mathrm{HbA} 1 \mathrm{c}, \%$ & $5.43(0.61)$ & $5.53(0.58)$ & 0.048 & $5.41(0.53)$ & $5.51(0.58)$ & 0.063 \\
\hline \multicolumn{7}{|l|}{ Lipid Profiles } \\
\hline Total cholesterol, mg/dL & $186.84(32.90)$ & $192.51(33.42)$ & $<0.001$ & $191.16(31.98)$ & $192.06(33.85)$ & 0.666 \\
\hline $\mathrm{HDL}, \mathrm{mg} / \mathrm{dL}$ & $54.07(13.97)$ & $56.10(14.68)$ & 0.006 & $55.42(14.75)$ & $55.76(14.58)$ & 0.713 \\
\hline $\mathrm{LDL}, \mathrm{mg} / \mathrm{dL}$ & $118.46(30.45)$ & $124.86(32.10)$ & $<0.001$ & $121.74(29.55)$ & $123.03(31.11)$ & 0.500 \\
\hline Triglyceride, mg/dL & $110.05(95.60)$ & $115.96(82.05)$ & 0.151 & $112.83(90.05)$ & $113.45(72.88)$ & 0.917 \\
\hline \multicolumn{7}{|l|}{ Life style } \\
\hline Smoking, \% & $11.0 \%$ & $17.0 \%$ & $<0.001$ & $16.5 \%$ & $17.3 \%$ & 0.736 \\
\hline Alcohol Consumption, \% & $7.7 \%$ & $18.3 \%$ & $<0.001$ & $16.1 \%$ & $15.7 \%$ & 0.863 \\
\hline Exercise, \% & $23.9 \%$ & $48.2 \%$ & $<0.001$ & $43.6 \%$ & $45.6 \%$ & 0.526 \\
\hline \multicolumn{7}{|l|}{ Cardiometabolic Outcomes } \\
\hline ATP III, number of MetS & $1.28(1.23)$ & $1.49(1.30)$ & $<0.001$ & $1.41(1.27)$ & $1.50(1.27)$ & 0.296 \\
\hline FRS, unit & $4.57(4.65)$ & $4.97(4.77)$ & 0.132 & $4.68(4.50)$ & $4.76(4.37)$ & 0.809 \\
\hline eGFR, mL/min per $1.73 \mathrm{~m}^{2}$ & 86.05 (19.43) & 84.30 (13.92) & 0.028 & $83.51(17.00)$ & 83.88 (13.79) & 0.704 \\
\hline
\end{tabular}

systolic and diastolic), though significantly larger BMI $(p=0.01)$ and waist circumference were observed at the follow up visit in the non-intervention group ( $p=$ 0.012 ) with a significantly decrease in waist circumference in the intervention group $(p<0.001)$. Further, there were higher blood glucose level observed in the non-intervention group at the follow up visit, together with a higher HbA1c level (all $p<0.05$ ). Furthermore, higher total cholesterol, LDL and triglyceride levels were observed at the follow up visit in the nonintervention group (both $p<0.05$ ). Compared to the non-intervention group at follow up visit, we demonstrated a significantly reduction in waist circumference, post-prandial glucose, and total cholesterol level, resulting in significantly decrease of ATP III $(-0.27 \pm 0.08$, $p=0.001)$ and a borderline improvement in eGFR.
Difference-in-difference analysis of various clinical parameters and associated cardiometabolic risks at follow up

As presented in Table 4, the result of the DID analysis for various variables before and after intervention observation period showed that there was substantial reduction in waist circumference, post-prandial glucose, total cholesterol, higher HDL (all $p<0.05$ ) and borderline reduction of BMI and LDL $(p=0.071 \& 0.082$, respectively) when comparing the intervention to the nonintervention group. As for life styles modification, we also demonstrated in the intervention group that exercise behavior significantly increased at the follow up visit ( $p=0.007$ within group), and showed significant differences when compared to the non-intervention group ( $p=0.014$ between groups). These changes of 
Table 2 The association between variables used to match with propensity score

\begin{tabular}{lccl}
\hline Variable & Coefficient & Standard error & $P$ \\
\hline Age & 0.003 & 0.008 & 0.649 \\
Male gender & 0.026 & 0.166 & 0.874 \\
Systolic blood pressure, mmHg & 0.002 & 0.005 & 0.743 \\
Waist Circumference, cm & -0.01 & 0.006 & 0.115 \\
Body mass index, kg/m2 & 0.007 & 0.025 & 0.790 \\
Fasting glucose, mg/dL & -0.001 & 0.005 & 0.802 \\
Total cholesterol, mg/dL & -0.016 & 0.010 & 0.100 \\
HDL, mg/dL & 0.016 & 0.009 & 0.077 \\
LDL, mg/dL & 0.017 & 0.010 & 0.086 \\
Triglyceride, mg/dL & 0.002 & 0.001 & 0.186 \\
Smoking & 0.069 & 0.185 & 0.710 \\
Alcohol & -0.063 & 0.186 & 0.735 \\
Exercise & 0.049 & 0.132 & 0.710 \\
\hline
\end{tabular}

Model fitness: $\mathrm{C}$ statistics $=0.549 P$ value of Hosmer and Lemeshow Goodness-of-Fit test $=0.311$

baseline variables between two groups over the observed study period in turn further resulted in substantially decrease of ATP III $(-0.36 \pm 0.06, p<0.001)$ and a borderline increase in $\operatorname{eGFR}(1.39 \pm 0.8, p=0.084)$ when the intervention group was compared to the nonintervention population.

\section{Discussion}

In the current study, we demonstrated that a strategic health promotion intervention based on HIP improved cardiovascular risks substantially in multiple dimensions, which included waist reduction, decrease in postprandial glucose, cholesterol level as well as ATP III score, which still holds true after DID analysis (HIP versus non-HIP). The intervention programs also successfully enhanced the level of physical activity, when combined with the positive effects of previous biochemical and anthropometric data, further resulted in substantial reduction of metabolic scoring, and a borderline improvement of renal function in terms of eGFR. These results indicate that our health improvement intervention provides evidence-based cardiometabolic risks improvement in an Asian population during the 3 years HIP in this study.

Owing to the rapid growth of several common risk factors responsible for large invisible epidemic chronic diseases burden including unhealthy diet, physical inactivity and tobacco use, the World Health Organization (WHO) had undertaken a series of programs and set a goal to reduce the morbidity and mortality from such diseases [13-15]. So far, it is well-known that both health promotion and health education programs from previous studies had successfully demonstrated the effectiveness on disease prevention or the cease of disease

Table 3 Difference between pre-test and post-test in each study group

\begin{tabular}{|c|c|c|c|c|c|c|c|c|c|}
\hline \multirow[b]{2}{*}{ Variable } & \multicolumn{3}{|c|}{ Non-intervention group $(n=502)$} & \multicolumn{3}{|c|}{ Intervention group ( $n=502)$} & \multicolumn{3}{|c|}{ Difference in posttest data } \\
\hline & Pretest & Posttest & $P^{a}$ & Pretest & Posttest & $p^{a}$ & $\overline{M e a n}$ & SE & $p^{\mathrm{b}}$ \\
\hline \multicolumn{10}{|l|}{ Physiologic parameters } \\
\hline Systolic blood pressure, mmHg & $117.03(13.75)$ & $117.54(14.85)$ & 0.426 & $117.57(14.67)$ & $116.78(13.94)$ & 0.191 & -0.76 & 0.91 & 0.403 \\
\hline Diastolic blood pressure, $\mathrm{mmHg}$ & $73.34(9.24)$ & $73.48(9.80)$ & 0.719 & $73.86(9.77)$ & $73.88(9.41)$ & 1.000 & 0.40 & 0.61 & 0.513 \\
\hline Waist circumference, $\mathrm{cm}$ & $80.5(9.3)$ & $80.98(9.06)$ & 0.012 & $80.5(9.8)$ & $79.51(9.69)$ & $<0.001$ & -1.46 & 0.61 & 0.016 \\
\hline Body mass index, $\mathrm{kg} / \mathrm{m}^{2}$ & $23.61(2.86)$ & $23.72(2.93)$ & 0.010 & $23.66(3.21)$ & $23.66(3.23)$ & 0.922 & -0.06 & 0.20 & 0.740 \\
\hline \multicolumn{10}{|l|}{ Serum sugar profiles } \\
\hline Fasting glucose, mg/dL & $94.22(16.95)$ & $95.95(17.65)$ & 0.001 & $94.22(13.54)$ & $95.12(14.43)$ & 0.079 & -0.83 & 1.02 & 0.419 \\
\hline Post-prandial glucose, mg/dL & $104.16(33.06)$ & $108.00(33.89)$ & 0.033 & $103.75(28.91)$ & $101.05(25.45)$ & 0.069 & -6.77 & 2.06 & 0.001 \\
\hline $\mathrm{HbA} 1 \mathrm{c}, \%$ & $5.41(0.53)$ & $5.69(0.63)$ & $<0.001$ & $5.51(0.58)$ & $5.72(0.70)$ & $<0.001$ & 0.04 & 0.06 & 0.524 \\
\hline \multicolumn{10}{|l|}{ Lipid Profiles } \\
\hline Total cholesterol, mg/dL & 191.16 (31.98) & $195.30(34.93)$ & $<0.001$ & $192.06(33.85)$ & $190.88(32.78)$ & 0.279 & -4.42 & 2.15 & 0.040 \\
\hline $\mathrm{HDL}, \mathrm{mg} / \mathrm{dL}$ & $55.42(14.75)$ & $56.10(15.49)$ & 0.110 & $55.76(14.58)$ & $55.21(14.52)$ & 0.039 & -0.90 & 0.98 & 0.362 \\
\hline $\mathrm{LDL}, \mathrm{mg} / \mathrm{dL}$ & $121.74(29.55)$ & $126.17(32.39)$ & $<0.001$ & $123.03(31.11)$ & $124.35(30.86)$ & 0.131 & -1.81 & 2.07 & 0.380 \\
\hline Triglyceride, mg/dL & $112.83(112.05)$ & $120.07(90.42)$ & 0.009 & $113.45(72.88)$ & $119.16(73.29)$ & 0.021 & -0.91 & 5.24 & 0.862 \\
\hline \multicolumn{10}{|l|}{ Cardiometabolic Outcomes } \\
\hline ATP III, number of MetS & $1.41(1.27)$ & $1.64(1.29)$ & $<0.001$ & $1.50(1.27)$ & $1.37(1.26)$ & 0.005 & -0.27 & 0.08 & 0.001 \\
\hline FRS, unit & $4.68(4.50)$ & $5.36(4.71)$ & 0.005 & $4.76(4.37)$ & $5.24(4.58)$ & 0.025 & -0.08 & 0.34 & 0.807 \\
\hline eGFR, mL/min per $1.73 \mathrm{~m}^{2}$ & $83.51(17.00)$ & $84.62(14.95)$ & 0.087 & $83.88(13.79)$ & 86.39 (14.64) & $<0.001$ & 1.72 & 0.94 & 0.068 \\
\hline
\end{tabular}

Data not specified were presented as Mean (SD); ${ }^{a}$ denotes paired-sample t-test; ${ }^{\text {b }}$ denotes independent-sample t-test 
Table 4 Difference in difference models with results displayed comparing Non-intervention and Intervention Groups

\begin{tabular}{|c|c|c|c|c|c|c|c|}
\hline \multirow[b]{2}{*}{ Variable } & \multicolumn{2}{|c|}{$\begin{array}{l}\text { Non-intervention group } \\
(n=502)\end{array}$} & \multicolumn{2}{|c|}{$\begin{array}{l}\text { Intervention group } \\
(n=502)\end{array}$} & \multicolumn{3}{|c|}{ Difference in difference (Intervention minus non-intervention) } \\
\hline & Mean & SD & $\overline{M e a n}$ & $S D$ & Mean & SE & $P^{a}$ \\
\hline \multicolumn{8}{|l|}{ Physiologic parameters } \\
\hline Systolic blood pressure, $\mathrm{mmHg}$ & 0.53 & 14.92 & -0.81 & 13.77 & -1.34 & 0.91 & 0.141 \\
\hline Diastolic blood pressure, $\mathrm{mmHg}$ & 0.16 & 10.08 & 0.00 & 9.73 & -0.16 & 0.63 & 0.795 \\
\hline Waist Circumference, cm & 0.72 & 5.95 & -0.96 & 6.04 & -1.69 & 0.4 & $<0.001$ \\
\hline Body mass index, $\mathrm{kg} / \mathrm{m}^{2}$ & 0.12 & 1.01 & 0.00 & 0.97 & -0.11 & 0.06 & 0.071 \\
\hline \multicolumn{8}{|l|}{ Serum sugar profiles } \\
\hline Fasting glucose, mg/dL & 1.72 & 11.06 & 0.92 & 11.70 & -0.79 & 0.72 & 0.271 \\
\hline Post-prandial glucose, mg/dL & 3.74 & 32.28 & -2.30 & 27.01 & -6.04 & 2.10 & 0.004 \\
\hline $\mathrm{HbA} 1 \mathrm{c}, \%$ & 0.26 & 0.33 & 0.22 & 0.62 & -0.05 & 0.05 & 0.315 \\
\hline \multicolumn{8}{|l|}{ Lipid Profiles } \\
\hline Total cholesterol, mg/dL & 4.13 & 23.92 & -1.08 & 22.16 & -5.21 & 1.47 & $<0.001$ \\
\hline $\mathrm{HDL}, \mathrm{mg} / \mathrm{dL}$ & -0.76 & 7.89 & 0.63 & 8.51 & 1.38 & 0.54 & 0.010 \\
\hline $\mathrm{LDL}, \mathrm{mg} / \mathrm{dL}$ & 3.85 & 22.95 & 1.40 & 19.96 & -2.45 & 1.41 & 0.082 \\
\hline Triglyceride, mg/dL & 7.17 & 60.98 & 5.43 & 52.17 & -1.74 & 3.61 & 0.630 \\
\hline \multicolumn{8}{|l|}{ Cardiometabolic Outcomes } \\
\hline ATP III, number of MetS & 0.23 & 1.02 & -0.12 & 0.96 & -0.36 & 0.06 & $<0.001$ \\
\hline FRS, unit & 0.55 & 3.49 & 0.41 & 3.24 & -0.15 & 0.27 & 0.582 \\
\hline eGFR, mL/min per $1.73 \mathrm{~m}^{2}$ & 1.10 & 14.24 & 2.48 & 10.71 & 1.39 & 0.80 & 0.084 \\
\hline
\end{tabular}

$S D=$ standard deviation; $S E=$ standard error of the mean difference; ${ }^{a}$ denotes independent-sample t-test

progression, with special focus on indices related to metabolic scorings which had been shown to be tightly linked to community health $[16,17]$. Tovar et al. had recently demonstrated that active control on diet contents may show beneficial effects on several cardiometabolic parameters including sugar level, several lipid profiles as well as blood pressure, which indicated the potentiality of dietary control based on the view points of primary preventive medicine [18]. Jahangiry et al. also ever published data regarding effects of lifestyle modification on metabolic syndrome in a dedicated designed randomized control trial, though in which study the case numbers are relatively small. In our current work, we similarly demonstrated that dietary modifications by active intervention showed positive effects on several lipid profiles, including drastic decline of total cholesterol, significant increase of HDL and borderline drop in LDL level, as well as improvement in post-prandial sugar control.

On the other hand, the significance of physical activity during lifestyle intervention had also been addressed and reported by Kujala et al., who observed a substantial decrease in both body weight and waist circumference for those at high risk for type 2 diabetes subjects but capable of carrying out higher physical activity level in the past 1 year [19]. The significant increase of exercise behavior and the substantial decrease of waist circumference and borderline decline of BMI at follow up visit in the HIP compared to the non-HIP group in our current study. In a previous report by Grundy et al., they showed that physical activity and weight reduction can act as first line therapy for metabolic syndrome [20, 21]. In our current work, we showed similar results in that HIP may effectively reduce metabolic scoring via increased physical activity and significantly reduced waist circumference, which was believed to be the key pathological component of metabolic syndrome. Our current data further extended beyond their findings in that we showed that these effects may be attributable to decreased total cholesterol, lowered post-prandial glucose as well as elevated HDL level.

Persistent physical training had been shown to be beneficial for prevention and disease management of diabetes and cardiovascular diseases [22-25]. Healthy diet controls, on the other hand, had also been shown to result in desirable health management results [26]. Of note, we showed that the clinical variables most subjective to the intervention therapy were waist circumference, body mass, post-prandial glucose level, renal function as well as several lipid profiles. There effects, when taken together, in part may help to explain the substantial improvement of metabolic scores at follow-up visit.

The strength of our work is the less biased nature of subjects enrolled for comparison between the HIP and 
non-HIP groups by utilizing propensity matching simulating randomized design from a relatively large Asian cohort since we fully acknowledged that potential selection bias in our work could not be avoided. The conduction of propensity matching may theoretically reduce the risks of baseline demographic distribution and the biochemical differences as much as possible. Furthermore, we conducted a propensity matching (PS) and difference-in-difference analysis (DID) which allows analysis of these effects less biased and to minimize the potential differences for those subjects with and without intervention during some period of follow-up. Even though, we still consistently showed the advantage of participating intervention programs in cardiometabolic risk reduction. To our best knowledge, there is a paucity of published studies that address relevant issues with relative large number of subjects enrolled from health evaluation center so far with meaningful results reported. While previous data on observational community-based reports seldom compare the potential effects of health promotion programs based on more strict study design with control group, our current data may provide invaluable information about the feasibility and usefulness of these programs implemented on a daily basis in tertiary care system $[18,27-30]$.

\section{Additional file}

Additional file 1: Table S1. The major components, content of examinations, measurements and intervention programs in Health Intervention Program (HIP) in our center. Figure S1. As shown in Figure S1, the age and sex distribution of all 3,057 individuals in this health screen program before the final enrollment criteria. Figure S2. Among 2,660 individuals who fit the enrollment criteria before propensity matching, 1,691 (63.6\%) were male and 969 (36.4\%) were female, resulting in a total of 922 and 1,738 subjects in the intervention and non-intervention groups, respectively. (DOC 232 kb)

\section{Competing interests}

The authors declare that they have no competing interests.

\section{Authors' contributions}

$\mathrm{CCL}$ participated in the manuscript drafting and conceptual frameworks. CLH carried out the statistical analysis and tables creations. SCS participated in study design and HJK conceived the study. REC participated the design and coordination in manuscript writing process. All authors read and approved the final manuscript.

\section{Acknowledgement \\ We also would like to express out gratefulness for Ming-Fei Tsai in the submission and reviewing of this work. This work was partially funded by grants from National Science Council (NSC-101-2314-B-195-020, NSC 103- 2314-B-010-005-MY3, 103-2314-B-195-001-MY3, 101-2314-B-195 -020 -MY1, MOST 103-2314-B-195-006-MY3), Mackay Memorial Hospital (10271, 10248, 10220, 10253, 10375, 10358, E-102003) and Taiwan Foundation for geriatric emergency and critical care.}

\section{Author details}

${ }^{1}$ The Institute of Health Policy and Management, College of Public Health, National Taiwan University, Taipei, Taiwan. ${ }^{2}$ Health Evaluation Center, Mackay Memorial Hospital, Taipei, Taiwan. ${ }^{3}$ Division of Cardiology, Department of
Internal Medicine, Mackay Memorial Hospital, Taipei, Taiwan. ${ }^{4}$ Division of Gastroenterology, Department of Internal Medicine, Mackay Memorial Hospital, Taipei, Taiwan.

Received: 30 March 2015 Accepted: 5 August 2015

Published online: 20 August 2015

\section{References}

1. Lloyd-Jones D, Adams R, Carnethon M, De Simone G, Ferguson TB, Flegal K, et al. "Heart Disease and Stroke Statistics 2009 Update: A report from the American Heart Association statistics committee and stroke statistics subcommittee". Circulation. 2009;119:480-6.

2. Pearson TA. New tools for coronary risk assessment: what are their advantages and limitations? Circulation. 2002;105:886-92.

3. Lakka H-M, Laaksonen DE, Lakka TA, Niskanen LK, Kumpusalo E, Tuomilehto $J$, et al. The metabolic syndrome and total and cardiovascular disease mortality in middle-aged men. JAMA. 2002;288:2709-16.

4. Ninomiya JK, L'Italien G, Criqui MH, Whyte JL, Gamst A, Chen RS. Association of the syndrome with history of myocardial infarction and stroke in the third National Health and Nutrition Examination Survey. Circulation. 2004;109:42-6.

5. Sattar N, Gaw A, Scherbakova O, Ford I, O'Reilly DS, Haffner SM, et al. Metabolic syndrome with and without C-reactive protein as a predictor of coronary heart disease and diabetes in the West of Scotland Coronary Prevention Study. Circulation. 2003;108:414-9.

6. Bonora E, Kiechl S, Willeit J, Oberhollenzer F, Egger G, Bonadonna RC, et al. Carotid atherosclerosis and coronary heart disease in the metabolic syndrome: prospective data from the Bruneck Study. Diabetes Care. 2003;26:1251-7.

7. Cupples LA, D'Agostino RB. Section 34: Some risk factors related to the annual incidence of cardiovascular disease and death in pooled biennial measurements. In: Kannel WB, Wolf PA, Garrison RJ, eds. Framingham Heart Study: 30 Year Follow-Up. Bethesda, MD: US Department of Health and Human Services (NIH publication). 1987;87:2703-7.

8. Woodward M, Brindle P, Tunstall Pedoe H, For the SIGN Group on Risk Estimation. Adding social deprivation and family history to cardiovascular risk assessment: the ASSIGN score from the Scottish Heart Health Extended Cohort (SHHEC). Heart. 2007;93:172-6.

9. Conroy RM, Pyorala K, Fitzgerald AP, Sans S, Menotti A, De Backer G, et al. Estimation of ten-year risk of fatal cardiovascular disease in Europe: the SCORE. Eur Heart J. 2003;24:987-1003.

10. Expert Panel on Detection, Evaluation, and Treatment of High Blood Cholesterol in Adults. Executive Summary of the Third Report of the National Cholesterol Education Program (NCEP) Expert Panel on Detection, Evaluation, and Treatment of High Blood Cholesterol in Adults (Adult Treatment Panel III). JAMA. 2001;285:2486-97.

11. Anderson KM, Wilson PW, Odell PM, et al. An updated coronary risk profile: a statement for health professionals. Circulation. 1991;83:356e362.

12. Levey AS, Bosch JP, Lewis JB, Greene T, Rogers N, Roth D. A more accurate method to estimate glomerular filtration rate from serum creatinine: a new prediction equation. Modification of Diet in Renal Disease Study Group. Ann Intern Med. 1999;130:461-70.

13. Resolution WHA57.17. Global Strategy on Diet, Physical Activity and Health. In: Fifty-seventh World Health Assembly, Geneva, 17-22 May 2004. Geneva, World Health Organization: Resolutions and decisions, annexes; 2004.

14. Preventing chronic diseases: a vital investment. Geneva, World Health Organization, 2005. http://www.who.int/chp/chronic_disease_report/ full_report.pdf.

15. Diet, nutrition and the prevention of chronic diseases. report of a joint WHO/FAO expert consultation. Geneva: World Health Organization; 2003. WHO Technical Report Series, No. 916.

16. WHO Regional Office for Europe. Ottawa Charter for health promotion. Geneva: WHO; 1986.

17. World Health Organization (WHO). New approaches to health education in primary health care. Geneva: WHO; 1983.

18. Tovar J, Nilsson A, Johansson M, Ekesbo R, Aberg AM, Johansson U, et al. A diet based on multiple functional concepts improves cardiometabolic risk parameters in healthy subjects. Nutr Metab. 2012;9:29.

19. Kujala UM, Jokelainen J, Oksa H, Saaristo T, Rautio N, Moilanen L, et al. Increase in physical activity and cardiometabolic risk profile change during lifestyle intervention in primary healthcare: 1-year follow-up study among individuals at high risk for type 2 diabetes. BMJ Open. 2011;1, e000292. 
20. Grundy SM, Hansen B, Smith Jr SC, Cleeman Jl, Kahn RA. American Heart Association, National Heart, Lung, and Blood Institute, American Diabetes Association: Clinical management of metabolic syndrome: report of the American Heart Association/National Heart, Lung, and Blood Institute/ American Diabetes Association conference on scientific issues related to management. Circulation. 2004;109:551-6.

21. Rennie KL, McCarthy N, Yazdgerdi S, Marmot M, Brunner E. Association of the metabolic syndrome with both vigorous and moderate physical activity. Int J Epidemiol. 2003;32:600-6.

22. Mohan V, Gokulakrishnan K, Deepa R, Shanthirani CS, Datta M. Association of physical inactivity with components of metabolic syndrome and coronary artery disease - The Chennai Urban Population Study (CUPS No. 15). Diabet Med. 2005;22:1206-11.

23. Knowler WC, Barrett-Connor E, Fowler SE, et al. Diabetes Prevention Program Research Group. Reduction in the incidence of type 2 diabetes with lifestyle intervention or metformin. N Engl J Med. 2002;346:393-403.

24. Ramachandran A, Snehalatha C, Mary S, Mukesh B, Bhaskar AD, Vijay V. Indian Diabetes Prevention Programme (IDPP). The Indian Diabetes Prevention Programme shows that lifestyle modification and metformin prevent type 2 diabetes in Asian Indian subjects with impaired glucose tolerance (IDPP-1). Diabetologia. 2006;49:289-97.

25. Kimura M, Moriyasu A, Kumagai S, Furuna T, Akita S, Kimura S, et al. Community-based intervention to improve dietary habits and promote physical activity among older adults: a cluster randomized trial. BMC Geriatr BMC Geriatrics. 2013;13:8

26. Traill WB, Shankar B, Brambila-Macias J, Bech-Larsen T, Aschemann-Witzel Strand $M$, et al. Interventions to promote healthy eating habits: evaluation and recommendations. Obes Rev. 2010;11(12):895-8.

27. Fukumoto K, Wei CN, Matsuo H, Harada K, Zhang SC, Kalay L, et al. An intervention study to promote self-improvement of lifestyle in a Japanese community: a new health support program. Environ Health Prev Med. 2011;16:253-63.

28. Kiyu A et al. Evaluation of the Healthy Village program in Kapit district Sarawak, Malaysia. Health Promot Int. 2006;21(1):13-8.

29. Ronda $\mathrm{G}$ et al. The Dutch Heart Health community intervention 'Hartslag Limburg': results of an effect study at individual level. Health Promot Int. 2004;19(1):21-31.

30. Mohan V et al. Community Empowerment? A successful model for prevention of non-communicable diseases in India? the Chennai Urban Population Study (CUPS-17). J Assoc Physicians India. 2006;54:858-62.

\section{Submit your next manuscript to BioMed Central and take full advantage of:}

- Convenient online submission

- Thorough peer review

- No space constraints or color figure charges

- Immediate publication on acceptance

- Inclusion in PubMed, CAS, Scopus and Google Scholar

- Research which is freely available for redistribution 